\title{
SignTalk and Animator for Speech and Hearing Imapired
}

\author{
Haseeb Biya ${ }^{1}$, Ruba Shaikh ${ }^{2}$, Sayma Siddiqui ${ }^{3}$, Sufiyan Khot $^{4}$ \\ Bachelor of Engineering, Computer Department, AIKTC, Panvel, India ${ }^{1,2,3,4}$
}

\begin{abstract}
Communication is basic fundamental human right, however who are deaf and mute communicate differently than everyone else using Sign Language (SL), while we communicate verbally. This puts them at disadvantage. Our system will help them better communicate with rest of the world without changing how they already interact with each other. The system i.e. Hand gloves will translate sign language to voice. flex sensors, accelerometer, gyroscope, are placed on hand gloves to capture hand movements. Arduino Nano recognizes these signals and sends them to smart phone via Bluetooth for voice generation. Animator is an android application that takes text sentences as input and converts it to 2D animations for facilitating two-way communication.
\end{abstract}

Keywords: Arduino Nano, Bluetooth Module, Accelerometer, Smart Phone, Flex Sensors, MPU60506-Axis Gyro Sensor Module, Android Studio, Sign Language(SL).

\section{INTRODUCTION}

The Deaf and mute community use Sign Language for conveying their thoughts. Sign Language involves simultaneous combining hand shapes, orientation and movement of hands, arms to express thoughts. While it's easy for them to communicate amongst themselves using sign language, the general public often finds it difficult to follow these gestures.

Interpreters are needed in such cases, It can be quite frustrating for them to constantly depend on interpreter whilst communicating with their peers. These People are treated differently.Thus creating a gap between normal people and them.Sign Language is not universal.

When India ratified the United Nations Convention on the rights of persons with disabilities (UNCRPD), India made a promise to the world that she would ensure that dumb and deaf people will be treated equally and will enjoy the same rights as other Indiancitizens.

But the absence of such a common sign language proves to be a hurdle in the efforts to treat the hearing and speech impaired people equally. As most of the people prefer cell phone for completing their chores the usage of smart phones has increased tremendously, thus use of cell phone as an interpreter makes it more portable and easy to use.

\section{PROJECT ARCHITECTURE}

The System Has Two Modules:

A. Sign Language Interpreter (Hand gloves).

B. Animator (Android application).

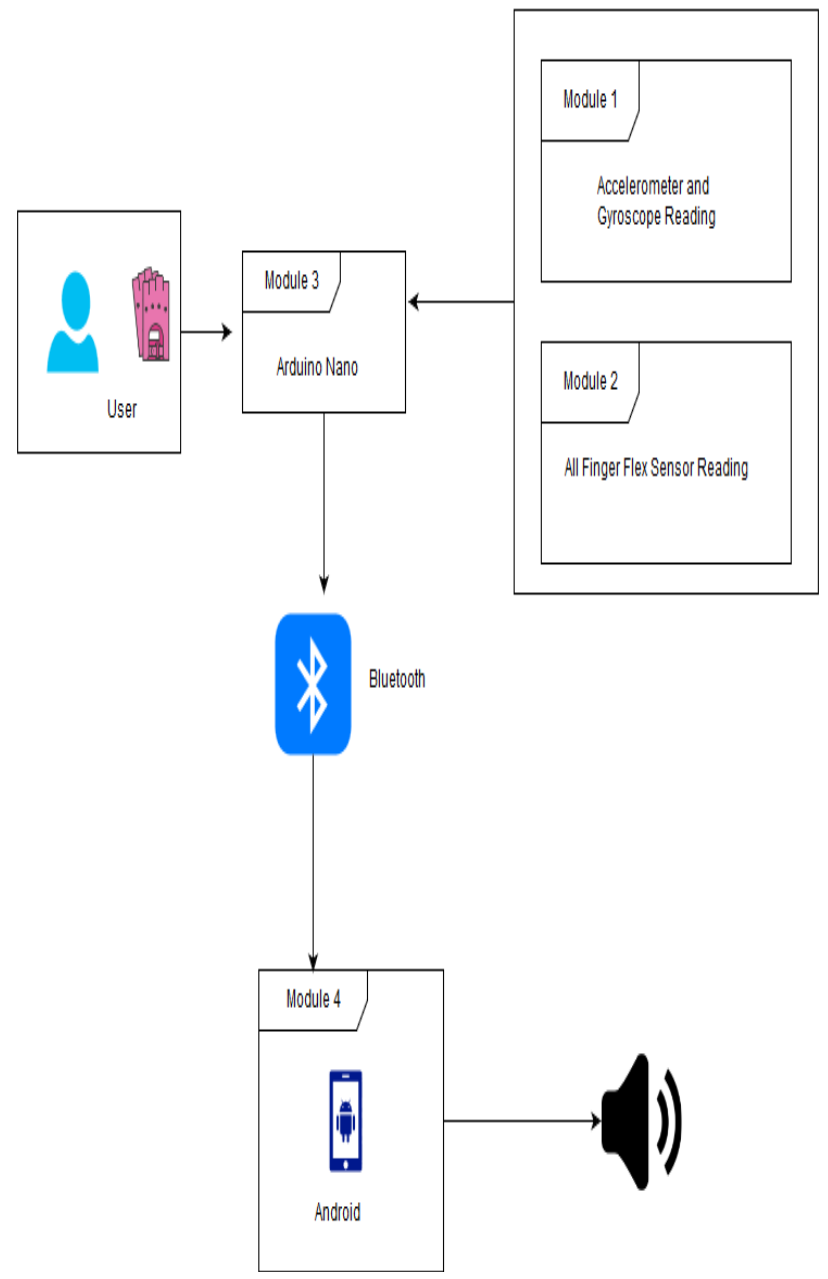

Fig. 1. Overall architecture of Interpreter

A. Sign Language Interpreter.

The overall system architecture is shown below:

B. Animator.

The overall system architecture is shown below: 


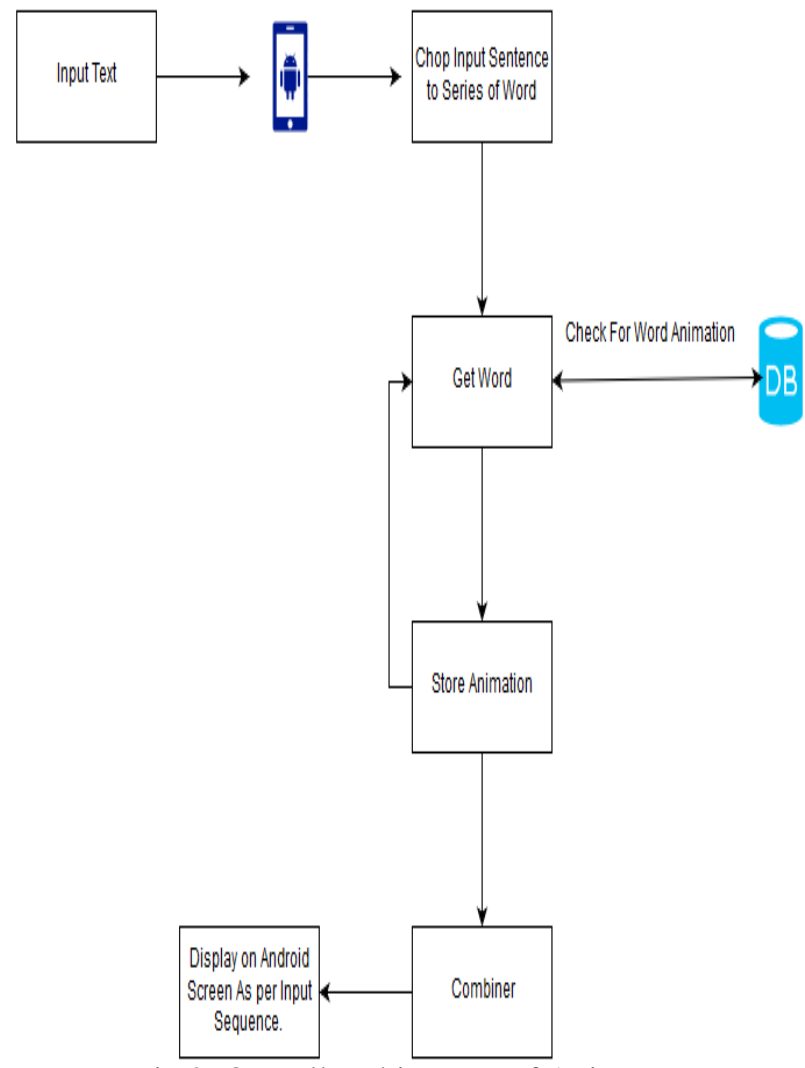

Fig.2. Overall architecture of Animator

\section{III.METHEDOLOGY}

A. SignTalk(Sign Language Interpreter).

SignTalk consists of sensors (flex, accelerometer, gyroscope) Arduino Nano and Bluetooth placed on the hand, then microcontroller converts the hand movements into audio. The device is built as a wearable glove, which converts the hand gestures into human recognizable audio. Flex sensors are placed on the glove measures finger bend, accelerometer measures the angle made by the arm with respect to earth, gyroscope measures the angular velocity of hand movement, all these analog signals are provided to an ATmega 328P microcontroller and are then converted to converted to digital values by $\mathrm{ADC}$ inbuilt in microcontroller. Microcontroller recognizes these values by comparing it to predefined range of values and sends it to smart phone via Bluetooth. Smart phone again identifies the data from Arduino and checks for its corresponding gestures values in database and plays the gesture audio accordingly.

Our System is divided into 4 modules. The components are as follows:

1. Accelerometer and Gyroscope module.

In this module data from accelerometer is captured in order to measure the angle made by hand with respect to earth. Gyroscope is used to measure the change in angular velocity. All this data is collected and given to Arduino Nano for further processing.

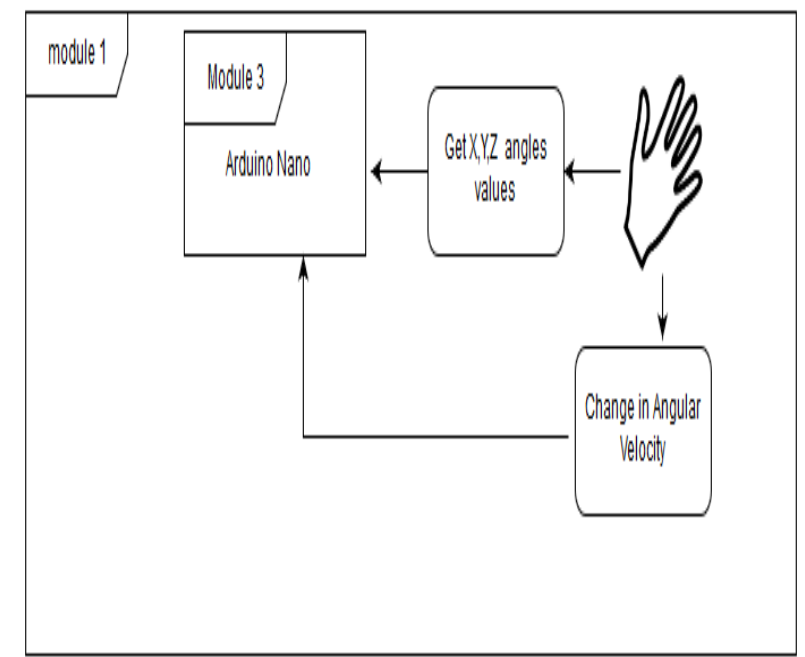

Fig. 3. Accelerometer and gyroscope module

2.All fingers flex sensor module.

In this module data from flex sensors placed over each finger is captured due to bending of finger. According to Ohm's Law V=IR as resistance changes there is change in voltage. Hence this change in voltage is given as an analog signal to Arduino Nano for further processing.

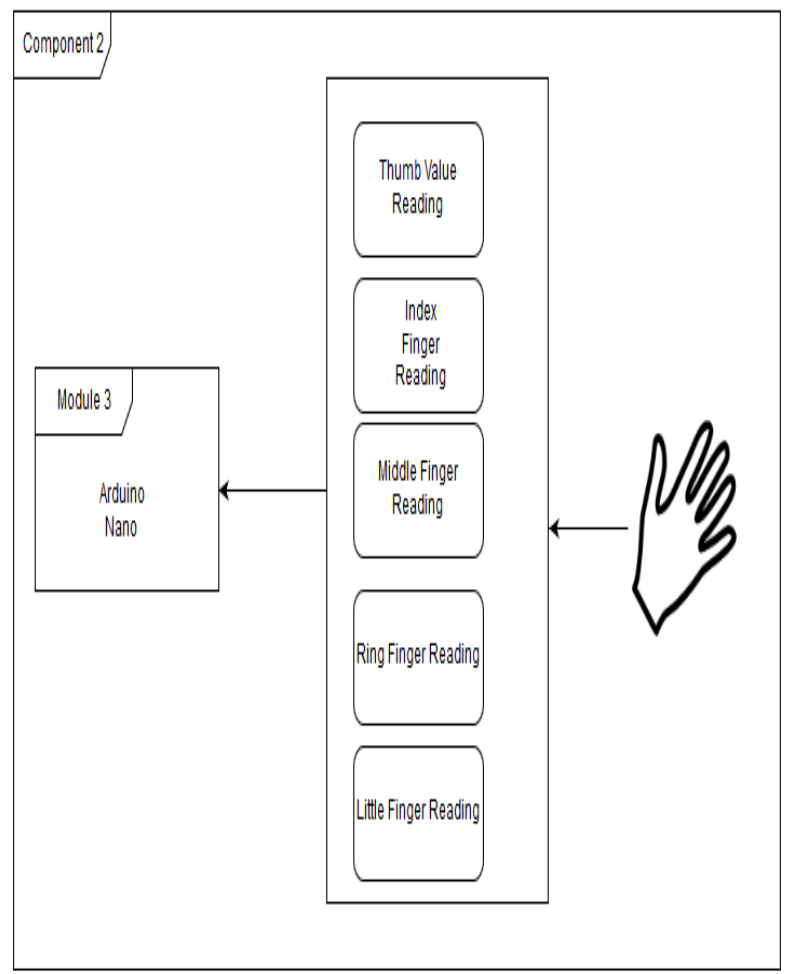

Fig. 4. Data flow in module 2.

3. Arduino Nano processing and recognition module.

In this module data from flex sensors, accelerometer and gyroscope is given to Arduino. Arduino matches these signals with pre-defined values and checks for any gesture in database. If found, then write the data on serial port with some special symbol as a terminator and is ready to transmit data to smart phone via Bluetooth. 


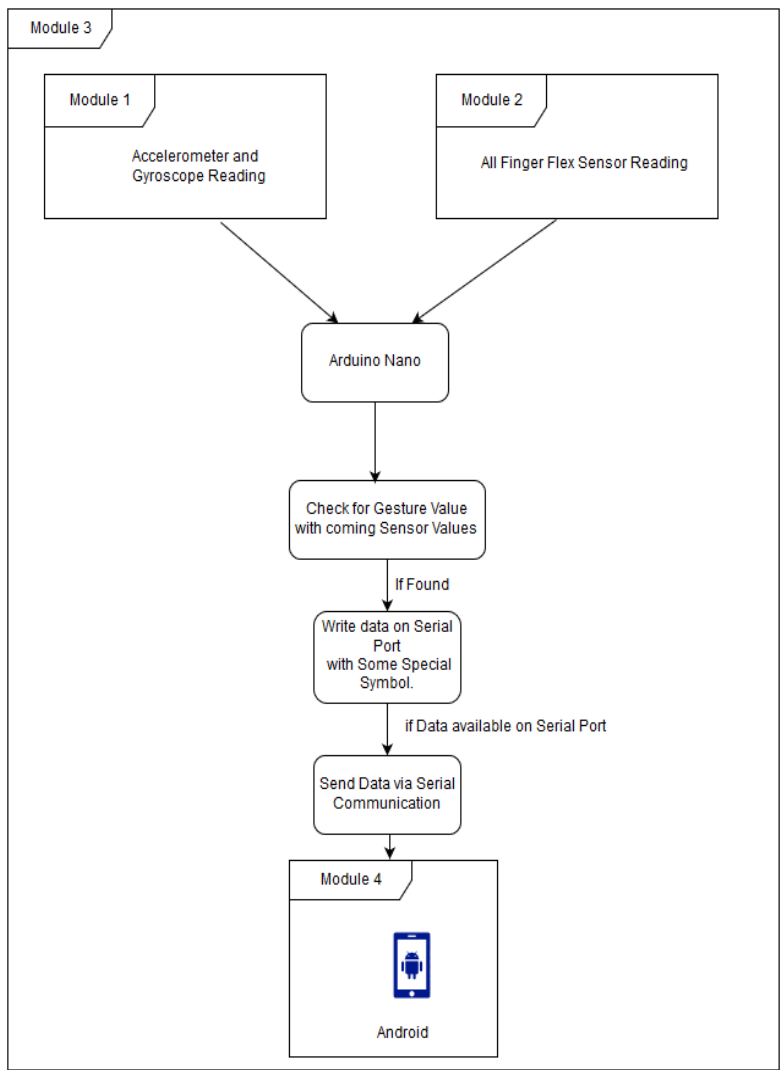

Fig.5. Data flow of module 3.

4. Android smart phone module.

In this module the Bluetooth continuously check for any data available in buffer. If found, then segregate and it checks for matching value audio and plays it.

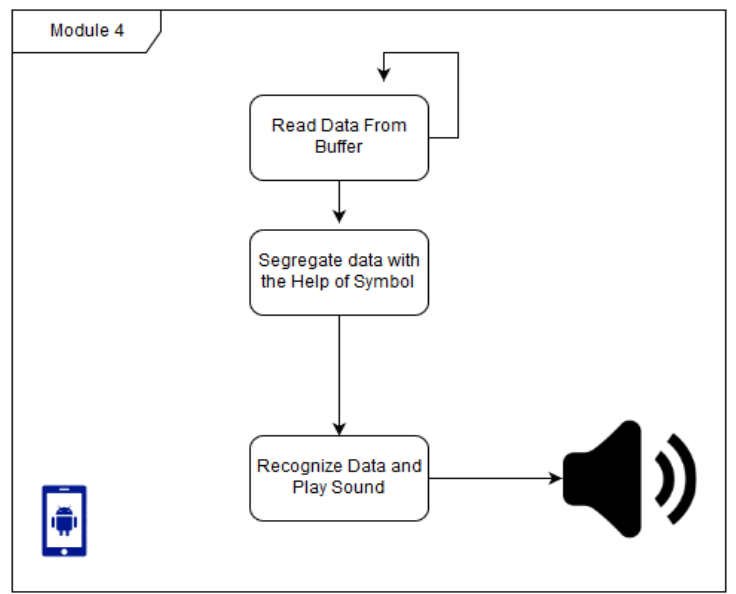

Fig.6. Data flow in module 4.

\section{B. Animator}

Animator provides two way communication. Animator can be used when an abled person wants to talk to a deaf and mute person. An abled person unknown of sign language can communicate with deaf and mute community using animator. The proposed application aims in providing an android based sign language synthesis output for the speech and hearing impaired. It based on the concept of
Flip Book animation. In this application user gives text input for which gesture can be generated. The input text is chopped and each frame corresponding to that word is fetched from database. All frames are fetched and then combined in same way as the input sequence. All frames are played giving a view of $2 \mathrm{D}$ animation. Moreover the application may be used as a teaching tool for relatives of deaf and dumb people as well as people interested in learning the SL.

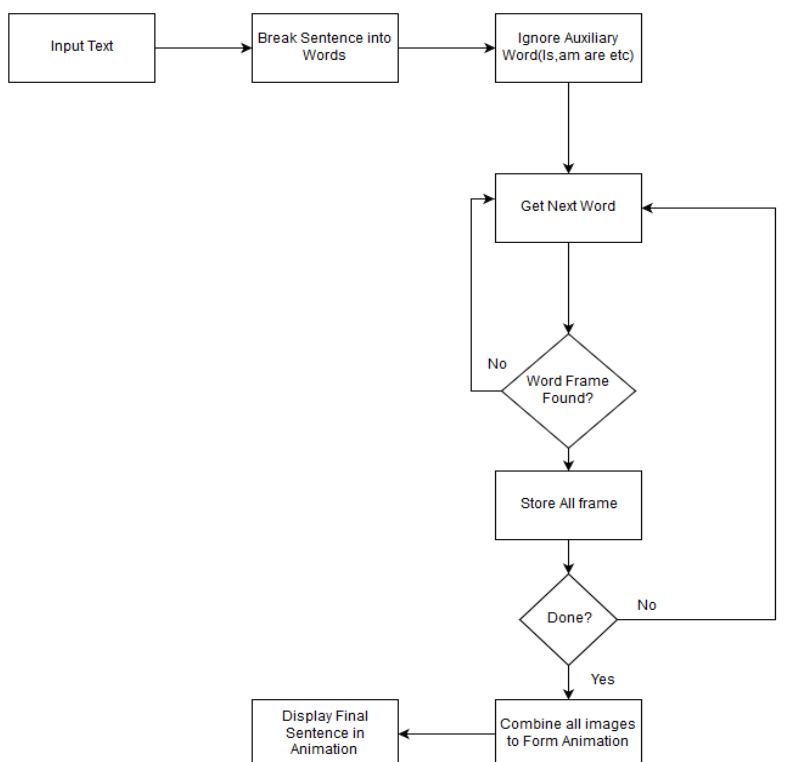

Fig.7. Animator flow chart

\section{Example:}

Suppose we want to convey: "WHAT IS YOUR NAME".As per algorithm the auxiliary words are removed. In this case "is" is an auxiliary word that gets filtered by the algorithm and left with "WHAT", "YOUR", "NAME". Each Word frame is fetched from database .Each frame is stored as per order of sentence. The stored frames are then given to the combiner which combines them and then plays the frames which appears to animate by simulating motion giving the view of $2 \mathrm{D}$ animation.

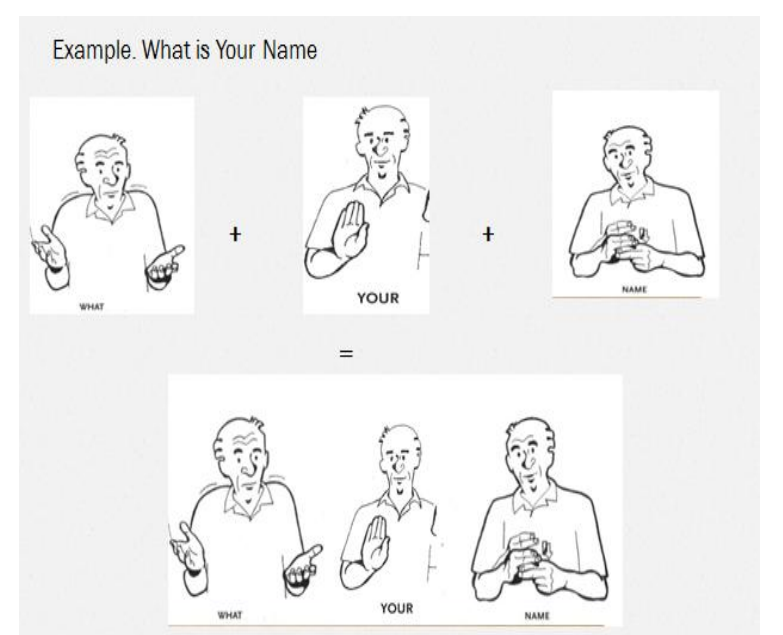

Fig. 8. Animator Example. 


\section{SOFTWARE HARDWARE REQUIREMENTS}

A. Arduino IDE:

The Arduino IDE is an Open-Source software used to provide instructions to microcontroller. It provides an environment where you can write code and upload it to your microcontroller. It is available for Windows, Linux and MAC operating system based computers.

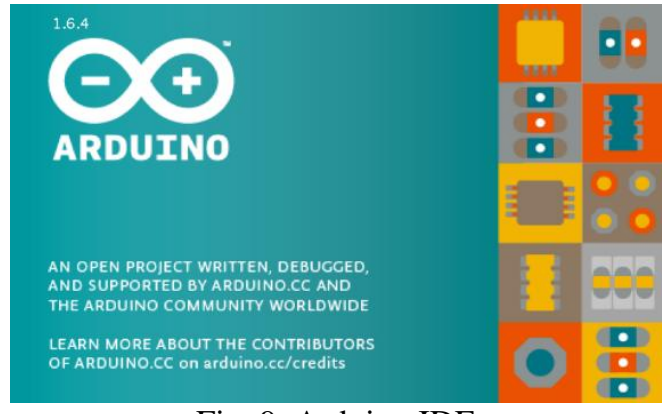

Fig. 9. Arduino IDE

B. Android Studio:

Android Studio is an Open-Source Integrated Environment for Developing Android Application.

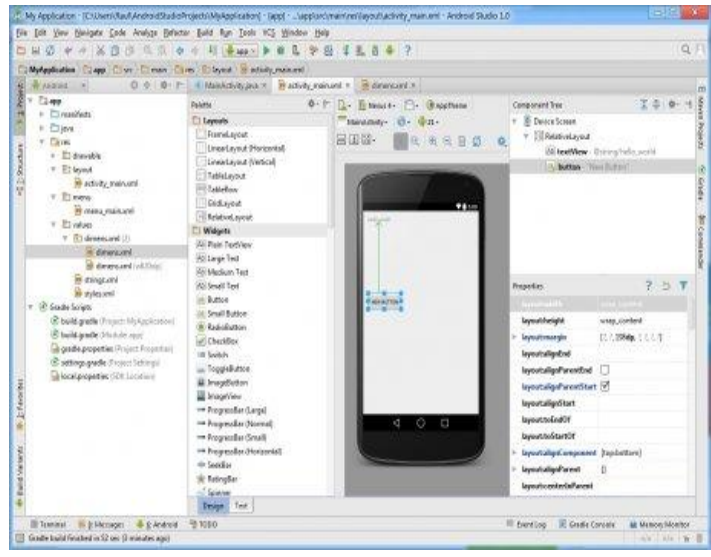

Fig. 10. Android Studio.

C. Flex Sensors:

Flex sensors are sensors that change resistance depending on the amount of bend on the sensor. They are analog sensors. Now the bending of each finger results in certain voltage drop this data from flex is sent to ATMEGA328P. Next step is to combine the movement of each finger and name it a particular gesture of the hand.

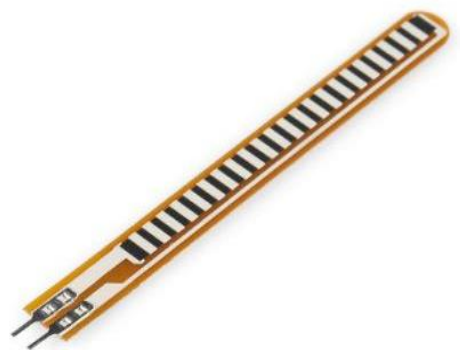

Fig. 11. flex sensor.

D. Accelerometer:

Accelerometer (ADXL 335) in the SignTalk system is used as a tilt detector. It has an analog output which varies from 1.5 volt to 3.5 volt.ADXL335 is a three-axis analog accelerometer IC, which reads off the $\mathrm{X}, \mathrm{Y}$ and $\mathrm{Z}$ acceleration as analog voltages. By measuring the amount of acceleration due to gravity, an accelerometer can figure out the angle it is tilted at with respect to the earth.

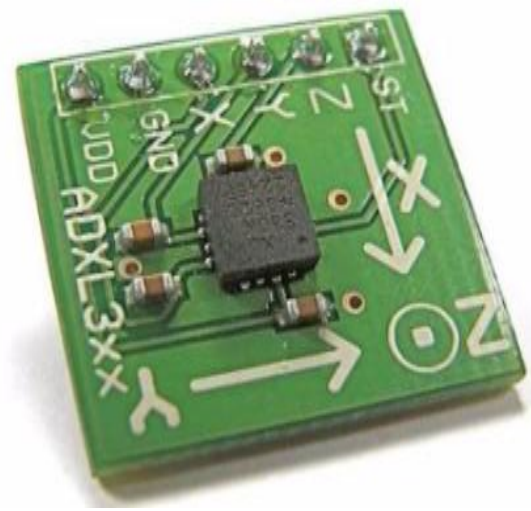

Fig.12. Accelerometer.

E. Gyroscope:

A device consisting of a wheel or disc mounted so that it can spin rapidly about an axis which is itself free to alter in direction. The orientation of the axis is not affected by tilting of the mounting, so gyroscopes can be used to provide stability or maintain a reference direction in navigation systems, automatic pilots, and stabilizers.

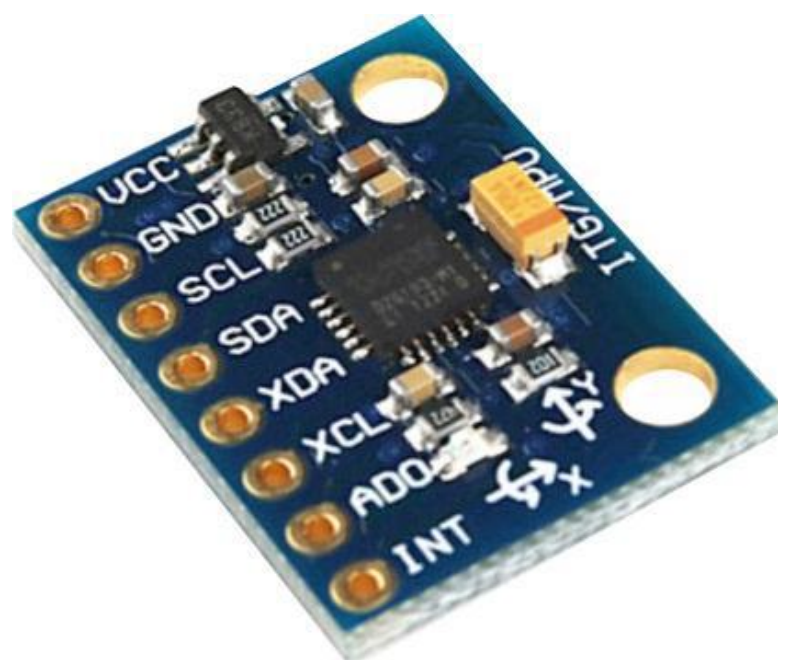

Fig.13. MPU6050 Accelerometer plus Gyroscope.

F. Arduino Nano:

Arduino Nano is a microcontroller board based on the ATmega328P. It has 14 digital input/output pins (of which 6 can be used as PWM outputs), 8 analog inputs, a $16 \mathrm{MHz}$ quartz crystal, a USB connection, a power jack. It contains everything needed to support the microcontroller. Simply connect it to a computer with a USB cable or power it with an AC-to-DC adapter or battery to get started. 


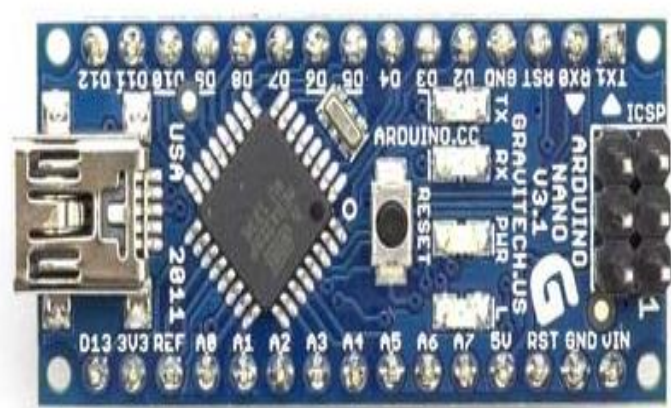

Fig. 14. Arduino Nano resolution

\section{G. Bluetooth:}

It captures the recognized signal from Arduino and sends it to smart phone for further processing and voice generation. Here HC-05 Bluetooth module is used.

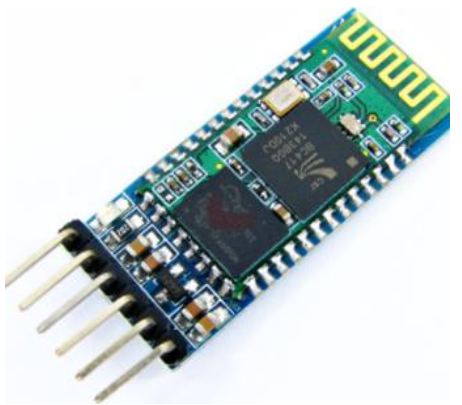

Fig. 15. Bluetooth

H. Smart phone:

It receives the signal via Bluetooth and compares it with the predefine values and generates audio accordingly. The android phone also compares the input text in the database and corresponding animation from database is fetched and combination of all broken text forms the sign language for the input.

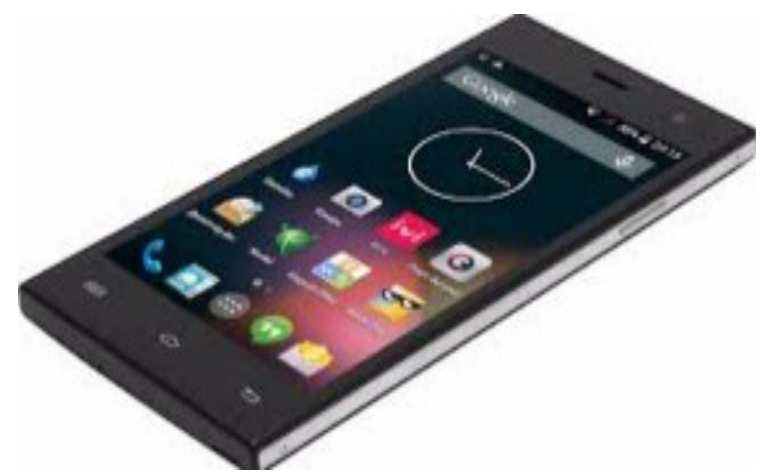

Fig. 16. Smart Phone

\section{IMPLEMENTATION}

\section{A. SIGNTALK(INTERPRETER)}

SignTalk is a Glove that is mounted on both the hands and has sensors over it.Depending upon the hand movements a Gesture is Generated and recognized by ArduinoThe Sensor on Hand Dynamically sets its value i.e. what we call as initialization. Initialization removes any absurd/incorrect values before using that SignTalk.If Initialization Failed, no Connection Will be Made.

Once Initialization is done now the user is allowed to perform Hand Movement that are in Strictly used Indian Sign Language. Arduino will Recognized and send data using Bluetooth.

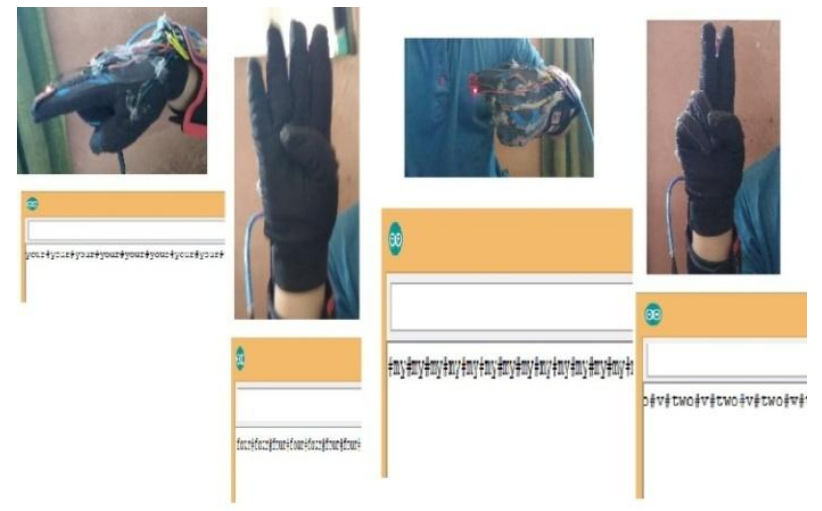

Fig.Testing

\section{B.Animator:}

We need to Provide Input of words/Sentences and then frames will be fetch from System and will be Displayed on Screen.

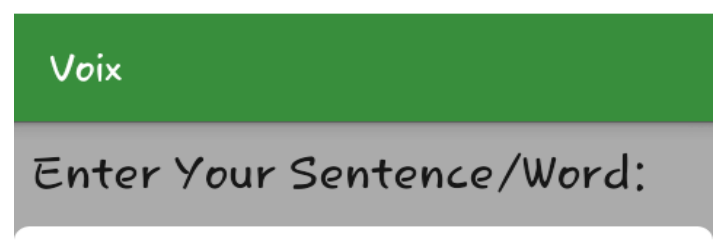

what is your name?

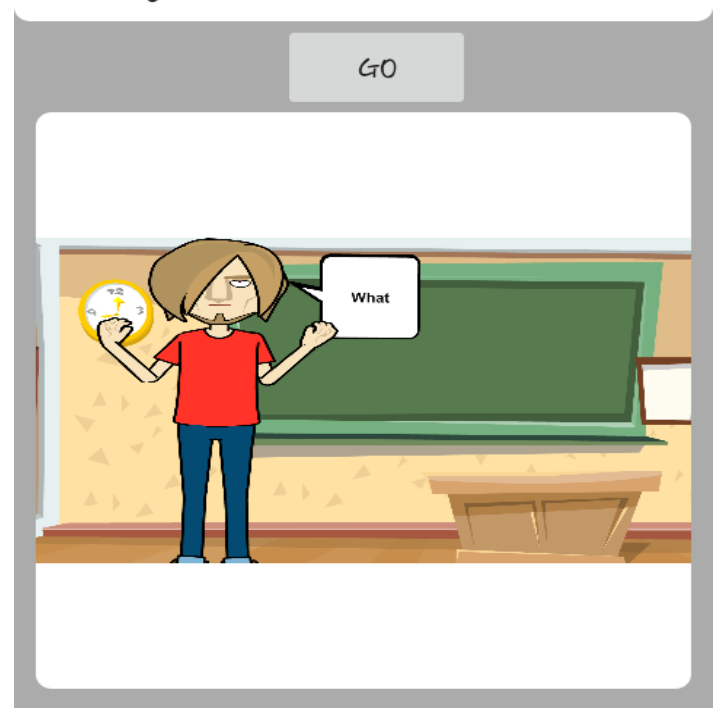

Fig.What 


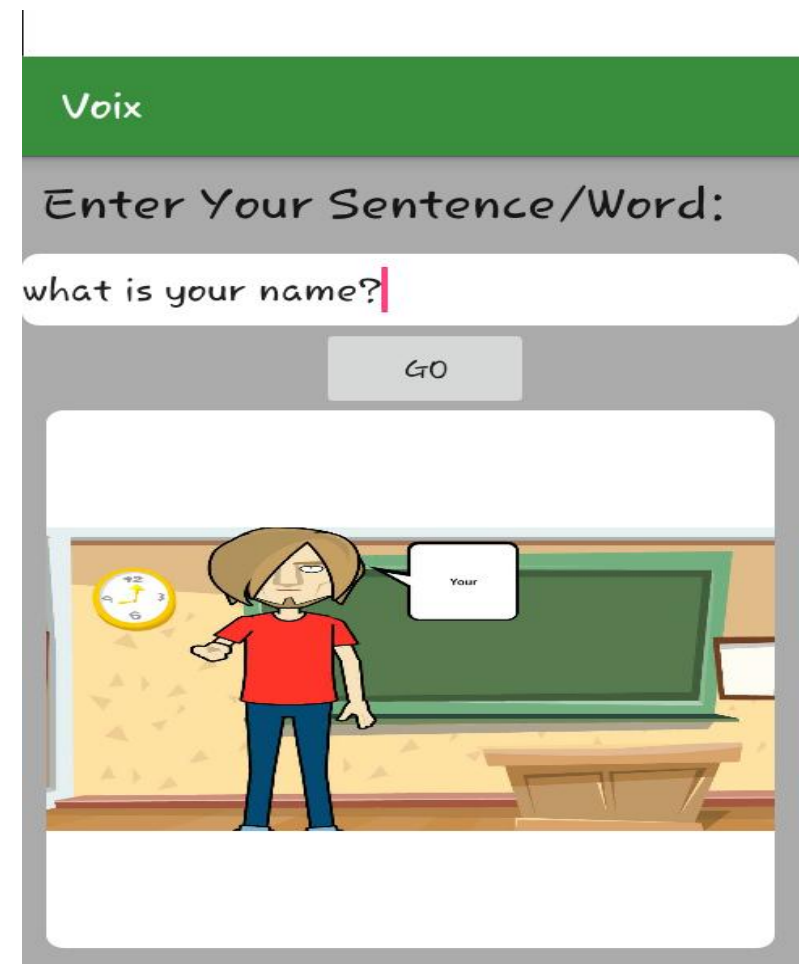

Fig.Your
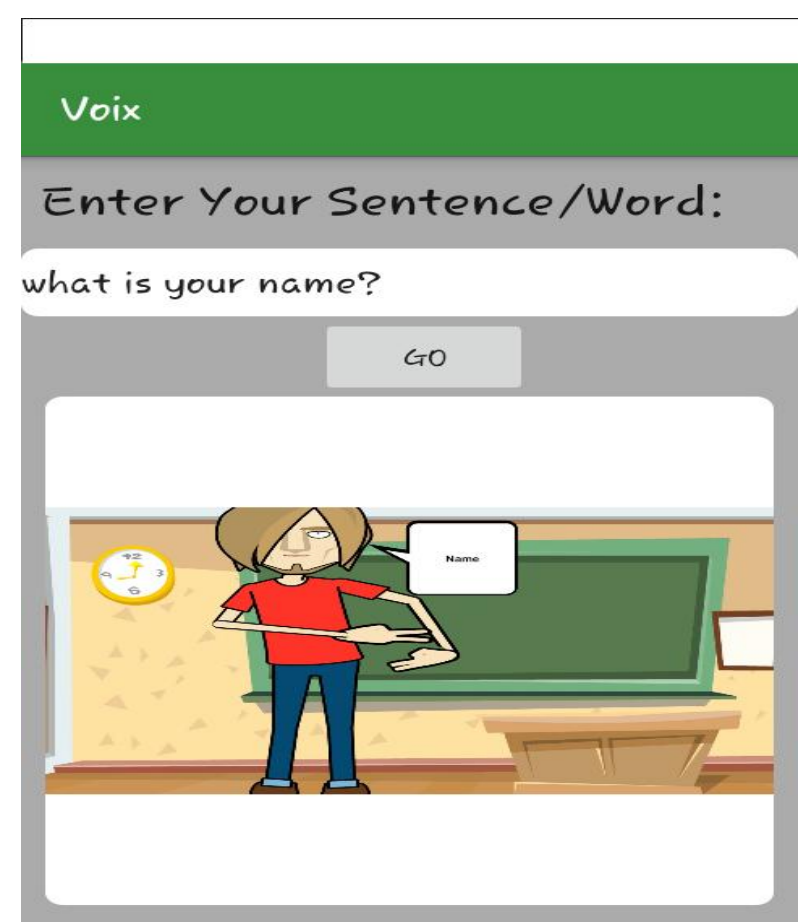

Fig.Name

\section{IMPLEMENTATION}

Sign language is a useful tool to ease the communication between the deaf or mute community and the normal people, yet there is a communication barrier between these communities with normal people as they have to depend on human translators. This project aims to lower the communication gap between the deaf and the normal world. With this project deaf and mute people can use the gloves while communicating so that hand movements will be converted into speech, understandable by others. There are over 70 million deaf and mute people in the world, our system will revolutionize the way they interact with others. With our system, they can achieve a new level of independence and an improved quality of life. In India There are about 1.5 million people using Sign Language, so it becomes very easy for them to convey almost all gestures with the gloves. To make it more acceptable in today's world we have provided an Android App which will act as an interpreter between them and us who are unaware of SL. Animator will help us to find appropriate SL while communicating to the deaf and mute. Also, in this project animator plays another important role in understanding and learning Sign Language. The project also provides two-way communication by mapping spoken language text to animation.

\section{REFERENCES}

[1] "Digital Vocalizer System for Speech and Hearing Impaired", by Kiran R Department of Electronics and Communication Engineering, College of Engineering Trivandrum, Thiruvananthapuram, India International Journal of Advanced Research in Computer and Communication Engineering Vol. 4, Issue 5, May 2015.

[2] "Electronic Hand Glove for Speech Impaired using MATLAB", by Nikita Ratnani, Mrs. Manisha Sharma, M.Tech Scholar , Department of ET\&T, Bhilai Institute of Technology, Durg, India1 Head of Department, Department of ET\&T,Bhilai Institute of Technology, Durg ,India International Journal of Advanced Research in Computer and Communication Engineering Vol. 5, Issue 4, April 2016.

[3] "Gesture Recognition System for Indian Sign Language on Smart Phone", by Ankit P. Parmar, Dr. Nehal G. Chitaliya, M.E Student, Dept. of Embedded System, Sardar Vallabhbhai Institute of Technology, Vasad-Gujarat, India1 Associate Professor, Dept. of Embedded System, Sardar Vallabhbhai Institute of Technology, Vasad-Gujarat, India. International Journal of Advanced Research in Computer and Communication Engineering Vol. 5, Issue 2, February 2016.

[4] "Gesture Based Vocalizer for Deaf and Dumb", by SupriyaShevate, Nikita Chorage, SiddheeWalunj, Moresh M. Mukhedkar Electronic and Telecommunication, Dr.D.Y.Patil College Of Engineering Ambi, TalegaonDabhade, Pune, India1,2,3M. Young, The Technical Writer's Handbook. Mill Valley, CA: University Science, 1989.International Journal of Advanced Research in Computer and Communication Engineering Vol. 5, Issue 3, March 2016.

[5] "Translating Indian Sign Language to text and voice messages using flex sensors" Sachin Bhat, Amruthesh M, Ashik, Chidanand Das, Sujith Asst. Professor, Dept. of E\&C, Shri MadhwaVadiraja Institute of Technology, Udupi, Karnataka, India1 UG Student, Dept. of E\&C, Shri MadhwaVadiraja Institute of Technology, Udupi, Karnataka, India. International Journal of Advanced Research in Computer and Communication Engineering Vol. 4, Issue 5, May 2015.

[6] "Sign Language to Speech Translation System Using PIC Microcontroller", by Gunasekaran. K1, Manikandan. R2 Senior Assistant Professor2 1,2School of Computing, SASTRA University, Tirumalaisamudram, Tamilnadu, India. Gunasekaran. $\mathrm{K}$ et al. / International Journal of Engineering and Technology (IJET).

[7] "Indian Sign Languages using Flex Sensor Glove", by Solanki Krunal Department of Biomedical Engineering, Govt. Engineering College, Gandhinagar, India International Journal of Engineering Trends and Technology (IJETT) - Volume4 Issue6- June 2013. 
[8] "Inertial Motion Sensing Glove for Sign Language Gesture Acquisition and Recognition", by Jakub Gałka, Member, IEEE, MariuszMa sior, Mateusz Zaborski, and KatarzynaBarczewska. IEEE SENSORS JOURNAL, VOL. 16, NO. 16, AUGUST 15, 2016.

[9] "Static and Dynamic Hand Gesture Recognition in Depth Data Using Dynamic Time Warping", by Guillaume Plouffe and AnaMaria Cretu, Member, IEEE.IEEE TRANSACTIONS ON INSTRUMENTATION AND MEASUREMENT, VOL. 65, NO. 2, FEBRUARY 2016.

\section{BIOGRAPHIES}

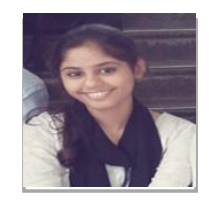

SaymaSiddiiqui, currently a final year student at AIKTC, Panvel, Mumbai University. She is pursuing Bachelor of Computer Engineering Degree.

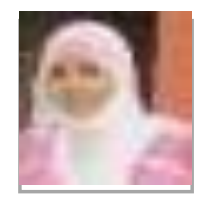

Ruba Shaikh, currently a final year student at AIKTC, Panvel, Mumbai University. She is pursuing Bachelor of Computer Engineering Degree.

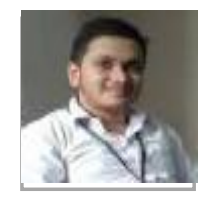

HaseebBiya, currently a final year student at AIKTC, Panvel, Mumbai University. He is pursuing Bachelor of Computer Engineering Degree.

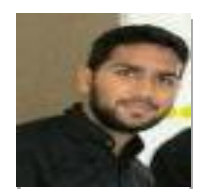

Sufiyan Khot, currently a final year student at AIKTC, Panvel, Mumbai University. He is pursuing Bachelor of Computer Engineering Degree. 